\title{
Study of structural short order and surface changes of $\mathrm{SiO}_{2}$ compounds
}

\author{
A. Oufakir ${ }^{1,2}$, L. Khouchaf ${ }^{2}$, M.Elaatmani ${ }^{1}$, A. Zegzouti ${ }^{1}$, G. Louarn ${ }^{3}$ and A. Ben Fraj ${ }^{4}$ \\ ${ }^{1}$ Laboratoire Sciences des Matériaux Inorganiques et leurs Applications, faculté des Sciences Semlalia, Université Cadi \\ Ayyad Marrakech -Maroc. \\ ${ }^{2}$ IMT Lille Douai, Lille Université, Cité Scientifique, Rue Guglielmo Marconi, BP 20145, 59653 Villeneuve D’Ascq \\ Cedex-France. \\ ${ }^{3}$ Institut des Matériaux Jean Rouxel, UMR 6502, Université de Nantes-CNRS, 2,14 Rue de la Houssiniére 44322, Nantes \\ Cedex, France. \\ ${ }^{4}$ Laboratoire Eco-Matériaux, CeremaIdF, 120 Route des Paris - BP 216, Sourdun, 77487 Provins Cedex, France
}

\begin{abstract}
In this work dependence between structure, surface and reactivity of $\mathrm{SiO}_{2}$ is investigated. Different samples are prepared with and without the presence of calcium cations and are characterized by different techniques. The FTIR frequency shifting of the bridging oxygen stretching vibration $\mathrm{Si}-\mathrm{O}-\mathrm{Si}$ and the binding energy shiftingof the Si2p and O1s photoelectron peaks have been observed and discussed. Results show the effect of presence of calcium on the structural change, surface and reactivity of $\mathrm{SiO}_{2}$ aggregate. After reaction, a structural and $\mathrm{SiO}_{2}$ surface changes occur, as the FTIR spectra illustrate depending on the presence of the calcium cations.
\end{abstract}

\section{Introduction}

The compounds based on $\mathrm{SiO}_{2}$, are of interest in several fields: as strengthening additives in the potter's clay materials, insulator in electronic compounds and in glass industry, concrete, Polymers... [1]. Reactivity of $\mathrm{SiO}_{2}$ depends on the chemical process that occurs between amorphous or poorly crystallized $\mathrm{SiO}_{2}$ and the matrix, in turn depends on the surface state of $\mathrm{SiO}_{2}$ particles [2-4]. $\mathrm{SiO}_{2}$ surface and short range order is more important in bioactive silica based materials which are characterized by an open structure which enables the accommodation of alkali and alkali-earthcations. The network modifiers such as $\mathrm{Ca}, \mathrm{K}, \mathrm{Na}$, provoke the disruption of the continuity of starting network dueto the breaking of some of the $\mathrm{Si}-\mathrm{O}-\mathrm{Si}$ bonds leading to the formation of non-bridging oxygen groups ( $\mathrm{Si}-\mathrm{O}-$ $\mathrm{NBO}$ ).This is the key step of the bioactive process, because the concentration of $\mathrm{Si}-\mathrm{O}-\mathrm{NBO}$ groups controls the dissolution rate of the silica through the formation of silanol groups at the glass surface [5].

Detailed knowledge of the atomic structure of $\mathrm{Si}-\mathrm{O}-\mathrm{Si}$ species and the silanols on surface of $\mathrm{SiO}_{2}$ compounds and the effect of calcium cations on their atomic structure are essential to understand the nature of fluids-silicates interactions and the dehydration processes in the composite materials such as nanocomposites and concrete [6]. The silanol groups $(\mathrm{SiOH})$ are the main surface reactive sites and their properties (nature, concentration, distribution, accessibility, etc.) will determine the chemical activity of the silica [7]. But there is a strong relationship between reactivity and the structural order of silica compounds [8-9].

The aim of this work is to study the dependence between structure, surface state and reactivity of silica for different heterogeneous $\mathrm{SiO}_{2}$ compounds with and without the presence of calcium during the reaction.

\section{Materials and Experimental}

Different type of $\mathrm{SiO}_{2}$ compounds have been investigated. The materials used in this work are siliceous natural aggregates (called $\mathrm{SiO} 2-1, \mathrm{SiO} 2-2$ and $\mathrm{SiO} 2-3$ ). XRD results show that all compounds are crystalline structure similar to quartz. The results are compared with amorphous a-SiO2 compound from alpfa Aesaras reference.

The experimental protocol was carried out as follows[10]:

The mix of crushed aggregate and calcium oxide $\mathrm{Ca}(\mathrm{OH})_{2}$ is introduced in the autoclavein an oven at $80^{\circ} \mathrm{C}$.After $30 \mathrm{~min}$ of preheating, $10 \mathrm{ml} \mathrm{KOH}$ solution of $0.79 \mathrm{~mol} / \mathrm{lare}$ added. The mix is then putted in an oven to developreaction under controlled temperature and reaction time. Afterreaction time, we put the autoclave in frozen waterduring $5 \mathrm{~min}$ to stop the reaction. The 
soluble reaction products areremoved by selective acid treatment and filtration. The acidattack is done using 250 $\mathrm{ml}$ cold $0.5 \mathrm{M} \mathrm{HCl}$ solution. The samples are dried by acetone and diethyl ether treatment after the filtration, then kept inside dried atmosphere.

The Transmission Electron Microscope investigations were performed with a FEI Tecnai G2-20 instrument with an accelerating voltage of $200 \mathrm{kV}$. It is equipped with a filament of lanthanum hexaboride LaB6, a double-tilt holder, an EDS analysis and Gatan digital camera CCD ORIUS.

The XPS measurements were performed using a spectrometer with a monochromatized AlK $\alpha$ X-ray source $(\mathrm{h} v=1486.6 \mathrm{eV})$. The data were collected on the dried and powdered samples which are pressed onto a substrate and is fixed on a sample holder. The samplesare introduced successively in a chamber at a constant pressure $\left(\mathrm{P}=10^{-3}\right.$ torr $)$ and in an analytical chamber $(\mathrm{P}=$ $10^{-9}$ torr). The ejected electronsphotoelectrons whose binding energies are inferior to the X-ray energy are collected according to their kinetic energies into a hemispherical $150 \mathrm{~mm}$ mean radius electron analyzer.

FTIR spectra were acquired with a Brucker VERTEX 70 spectrometer in reflection mode. They were recorded by collecting 100 scans at $4 \mathrm{~cm}^{-1}$ resolution in the range of $400-5000 \mathrm{~cm}^{-1}$.

\section{Results and discussion}

Figure 1 shows an image of the sample SiO2-1. Grains of different sizes ranging from ten nanometers to a few micrometers, with angular sides characterizing angles of quartz can be seen. Moreover, other areas of a rather disordered or amorphous appearance are observed. The samples $\mathrm{SiO} 2-2$ and $\mathrm{SiO} 2-3$ are the same appearance except $\mathrm{SiO} 2-3$ which presents more heterogeneous aspect.

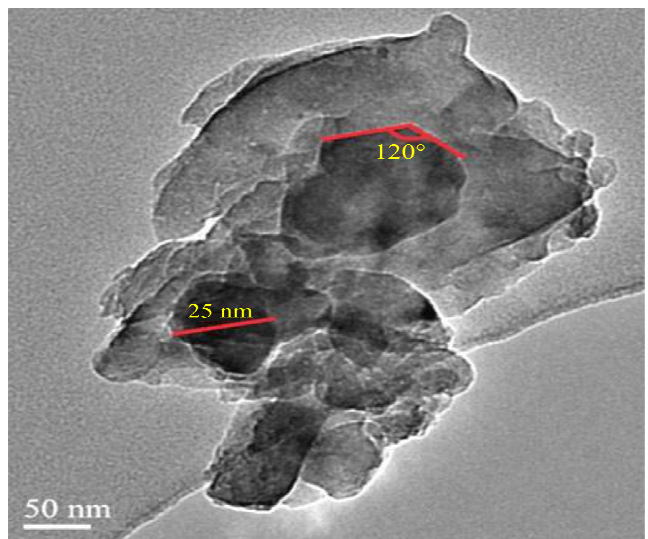

Fig. 1. Transmission Electron Microscope image of SiO2-1 sample.
Analysis of different zones of the samples shows the presence of silicon and oxygen elements as shown in Figure 2. Carbon and copper presence is attributed to the sample holder.

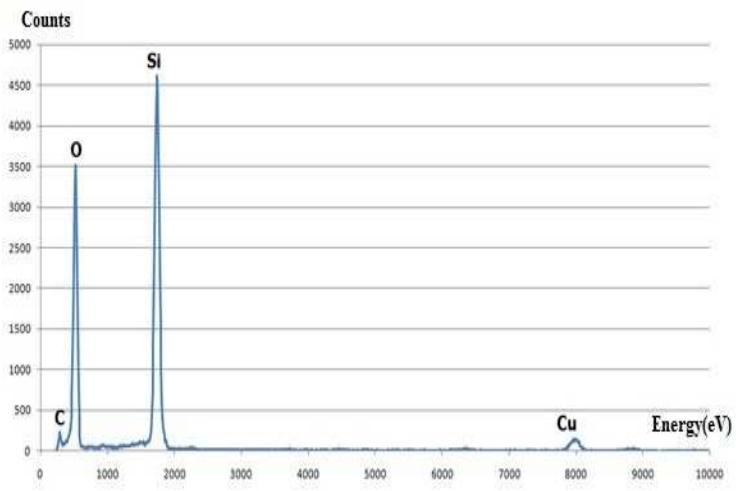

Fig.2. Energy dispersive spectrum of $\mathrm{SiO} 2-1$

In order to follow the reactivity of each aggregate, we subjected them to reaction according to the procedure described above. Figure 3 shows the results of the mass recovered after the reaction in the absence of calcium. It is observed that the mass recovered after reaction of the amorphous silica is the lowest, which confirms the expected results since it has the most disordered structure and therefore is the most reactive. In fact, the samples can then be classified in order of increasing reactivity, ranging from $\mathrm{SiO} 2-2, \mathrm{SiO} 2-1, \mathrm{SiO} 2-3$ to a-SiO2. These results are in good agreement with those obtained by $\mathrm{X}$ ray diffraction [8].In the presence of calcium, the recovered masses are most important, which shows the reactivity of these compounds decreases in the presence of calcium in agreement with previous study [10].

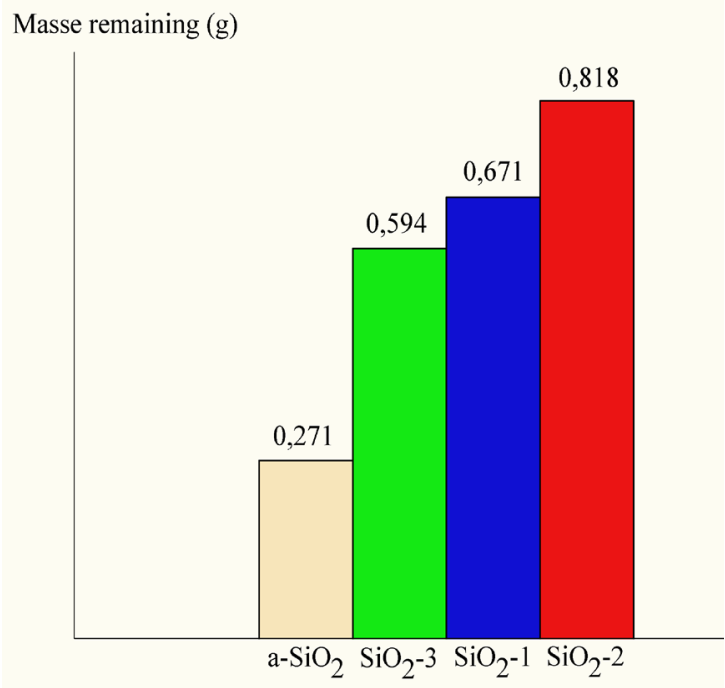

Fig. 3. Evolution of the mass remaining after reaction without calcium. 
In order to follow the evolution of the structure as well as the state of the surface, IR spectra of the different samples are recorded. The $\mathrm{SiO} 2-1$ as an intermediate case is showed as example.

Figure4 shows the FTIR spectra for SiO2-1 after reaction with and without the presence of calcium compared to SiO2-1 before reaction as reference. These FTIR spectra present the surface species as indicated in the figure.

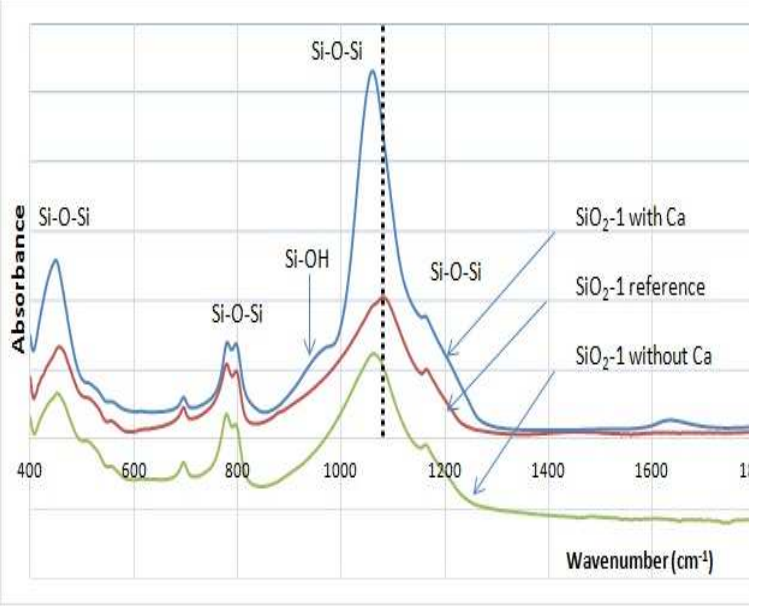

Fig.4. FTIR spectra of $\mathrm{SiO} 2-1$ after reaction with the presence and the absence of calcium compared to $\mathrm{SiO} 2-1$ reference before reaction.

The infrared spectra of all samples have a broad band between $1000 \mathrm{~cm}^{-1}$ and $1300 \mathrm{~cm}^{-1}$. This band consists of a strong peak. The mean peak is associated with the stretching vibration of Si-O-Si [11-12]. In addition, different bending vibrations attributed to $\mathrm{Si}-\mathrm{O}-\mathrm{Si}$ [13-15] are indicated in the figure. The bands located at $555 \mathrm{~cm}^{-1}$ and $950 \mathrm{~cm}^{-1}$ are associated with $\mathrm{Si}-\mathrm{O}$ bending vibrations of no bridging $\mathrm{Si}-\mathrm{OH}$ bonds and stretching vibration respectively $\mathrm{Si}$ [13-15].

In addition, we can notice that the mean band of SiO2-1 spectra after reaction with and without the presence of calcium is shifted compared to $\mathrm{SiO} 2-1$ before reaction as reference. This pronounced shift may be connected to the structural order change of $\mathrm{SiO}_{2}$ [13]. Other structural change of $\mathrm{Si}-\mathrm{O}-\mathrm{Si}$ is provided by the band at $800 \mathrm{~cm}^{-}$ ${ }^{1}$ (Figure5).

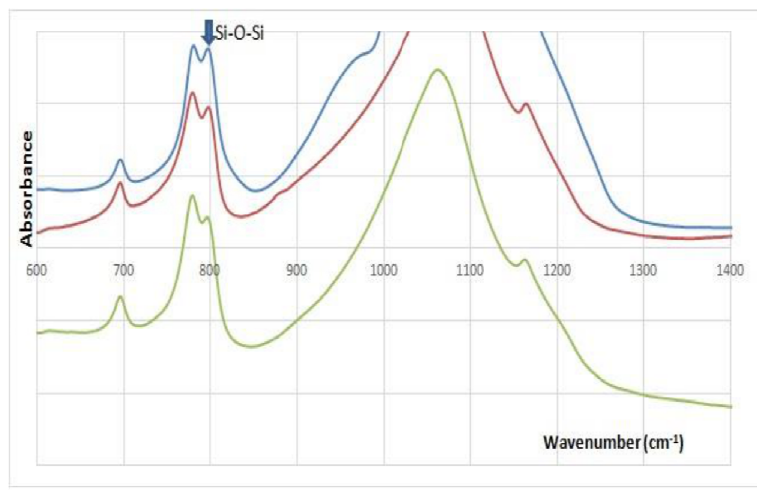

Fig.5. FTIR spectra of $\mathrm{SiO} 2-1$ after reaction with the presence and the absence of calcium compared to $\mathrm{SiO} 2-1$ reference before reaction.

Furthermore, it can be seen that $\mathrm{SiO} 2-1$ after reaction in the presence of calcium has a more intense peakassociated to $\mathrm{Si}-\mathrm{OH}$ groups and located at $950 \mathrm{~cm}^{-1}$ compared to $\mathrm{SiO} 2-1$ before reaction as reference and after reaction without calcium. The peak height from silanols groups increases in $\mathrm{SiO} 2-1$ with presence of calcium in comparison of $\mathrm{SiO} 2-1$ reference and $\mathrm{SiO} 2-1$ without the presence of calcium. This result shows that the surface state of $\mathrm{SiO} 2-1$ with the presence of calcium is different compared to $\mathrm{SiO} 2-1$ reference and $\mathrm{SiO} 2-1$ without the presence of calcium.

The XPS spectra were carried out to characterize the surface compositions of samples. Figure 6 presents O1s and $\mathrm{Si} 2 \mathrm{pXPS}$ spectra of $\mathrm{SiO} 2-1$ after reaction with the presence and the absence of calcium compared to $\mathrm{SiO} 2-1$ reference before reaction. Major elements detected are silicon and oxygen for all the samples.

Samples exhibit a clear binding energy difference between the $\mathrm{Si} 2 \mathrm{p}$ and $\mathrm{O} 1 \mathrm{~s}$ lines. In addition, the relative intensities of the Si2p peak and of the O1s peak vary from one sample to another; i.e., the samples do not display the same $\mathrm{Si} / \mathrm{O}$ atomic ratio. These features evidence that the chemical environment of siliconand oxygen is significantly affected by the presence of calcium more than the SiO2-1 sample with absence of calcium.

It is important to notice that the results represent typically the surface difference between the different samples confirming the changes and the results obtained with FTIR as explained before. 

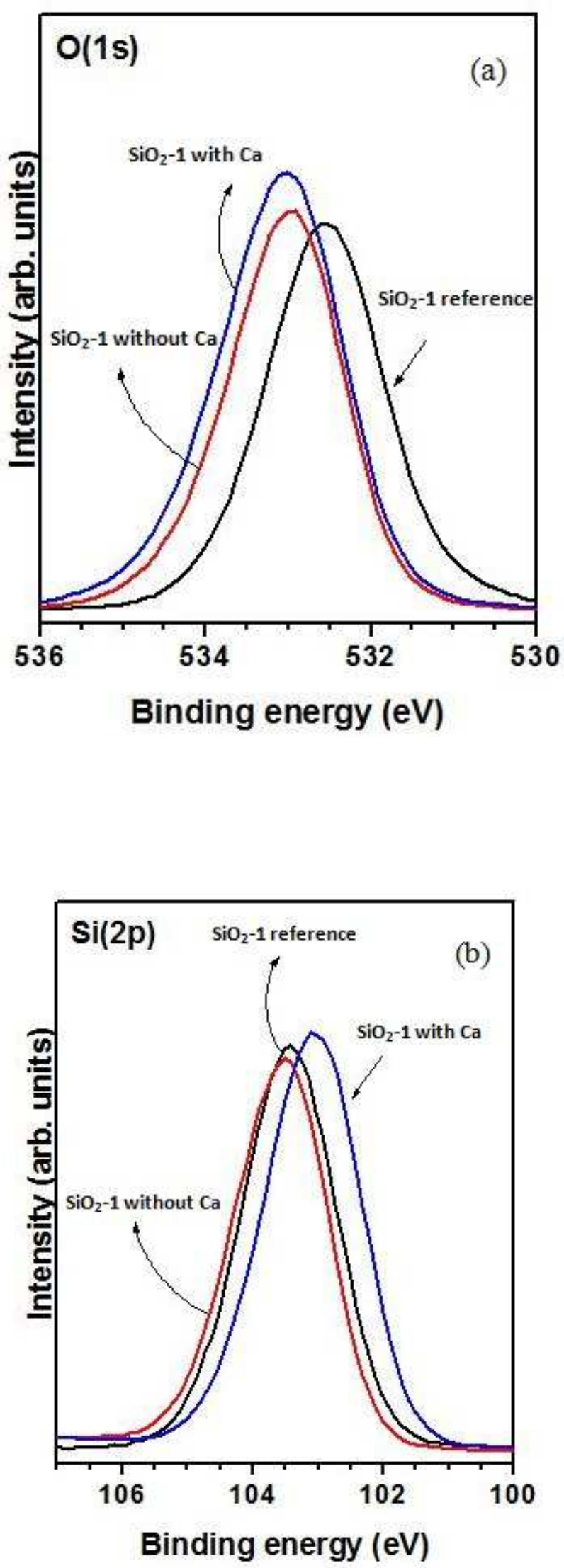

Fig.6. XPS spectra of SiO2-1 after reaction with the presence and the absence of calcium compared to $\mathrm{SiO} 2-1$ reference before reaction, (a) $\mathrm{O} 1 \mathrm{~s}$, (b) $\mathrm{Si} 2 \mathrm{p}$
The O1s photoelectron spectra for the samples are shown in Fig. 6a. The O1s signal is expressed by a symmetric function and it shifts towards lower energies with the presence of calcium. A similar behavior has been observed for the Si2p photoelectron peak, but shifts towards higher energies as shown in Fig. 6b typically with the presence of calcium.

Crystallized $\mathrm{SiO}_{2}$ samples are formed by an ordered network with perfect $\mathrm{SiO} 4$ tetrahedral connected to the adjacent tetrahedral by bridging oxygen atoms to form a three-dimensional connected network. Consequently, the contribution of $\mathrm{Si}-\mathrm{OH}$ infrared band is negligible as shown in Figure4. The presence of calciumwithSiO during the reactionchanges the degree of connectivity in the silicon- oxygen disordered network. In fact, the short range structural modifications occur as can be successfully detected by FTIR and XPS spectra.

In fact, the pronounced shift of the position of the peak and the increase of silanols surface species with the presence of calcium show a simultaneous modification of the structure and the surface of the starting aggregate after reaction. These results are interesting in a future study concerning the improvement of the interface properties of some composites materials with the presence of different silica compounds.

\section{Conclusion}

Dependence between structure, surface and reactivity of $\mathrm{SiO}_{2}$ is investigated. Different $\mathrm{SiO} 2$ samples with and without the presence of calcium cations are prepared and characterized by different techniques. Based on FTIR and XPS spectroscopy, frequency shifting of the bridging oxygen stretching vibration $\mathrm{Si}-\mathrm{O}-\mathrm{Si}$ and the binding energy shifting of the Si2p and O1s photoelectron peaks have been observed and discussed. Results show the effect of presence of calcium on the structural change, surface and reactivity of $\mathrm{SiO}_{2}$ aggregate. After reaction, a structural order and $\mathrm{SiO}_{2}$ surface changes occur, as the FTIR spectra illustrate depending on the presence of the calcium cations. TGA/TD and NMR experiments are planned in order to obtain more details about the silanols behavior.

This research was supported by European FEDER and region Nord-Pas-de-Calais.

\section{References}

1. T. H. Zhou, W. H. Ruan, J. L. Yang, M. Z. Rong, M. Q. Zhang, Z. Zhang, A nouvel route for improving creep resistance of polymers using nanoparticles, Composites Science and Technology, 21, 539 (2007).

2. F. Boinski, L. Khouchaf, and M-H. Tuilier, Study of the mechanisms involved in reactive 
silica, Materials Chemistry and Physics, 122, 311-315, (2010).

3. L. Khouchaf, A. Hamoudi and P. Cordier Evidence of depolymerisation of amorphous silica at medium and short range order: XANES, NMR and CP-SEM contributions, Journal of $H$. Materials, 168, 1188, (2009).

4. A. Hamoudi, L. Khouchaf, C. Depecker, B. Revel, L. Montagne, P. Cordier, « Microstructural evolution of amorphous silica following Alkali-Silica Reaction 》Journal of non Cryst. Solids, 354, 45-46 5074, (2008).

5. T. Kokubo, in: T. Yamamuro, L.L. Hench, J. Wilson (Eds.), Handbook of Bioactive Ceramics, CRC, Boca Raton, FL, p. 41,(1990).

6. Qiang Huang, Meiying Liu, Liucheng Maoa, Dazhuang Xu, Guangjian Zeng, Hongye Huang, Ruming Jiang, Fengjie Deng, Xiaoyong Zhang, Yen Wei, Surface functionalized SiO2 nanoparticles with cationic polymers via the combination of mussel inspired chemistry and surface initiated atom transfer radical polymerization: Characterization and enhanced removal of organic dye, Journal of Colloid and Interface Science, 499, 170-179, (2017).

7. Jean-Paul Gallas, Jean-Michel Goupil, Alexandre Vimont, Jean-Claude Lavalley, Barbara Gil, Jean-Pierre Gilson, and Olivier Miserque, Quantification of Water and Silanol Species on Various Silicas by Coupling IR Spectroscopy and in-Situ Thermogravimetry, Langmuir, 25,5825-5834, (2009)

8. H. El Bahraoui, L. Khouchaf, A. Ben Fraj, "Microscopical and mechanical evaluation of the durability of $\mathrm{SiO} 2$ aggregates", European Physical Journal of Applied Physics,74, 2, (2016).

9. N. Tahiri, L. Khouchaf, M. Elaatmani, G. Louarn, A. Zegzouti and M. Daoud, "Study of the thermal treatment of $\mathrm{SiO} 2$ aggregate," IOP Conf. Series: Materials Science and Engineering, vol. 62, doi:10.1088/1757899X/62/1/012002, (2014).

10. J. Verstraete , L. Khouchaf, and M. H.Tuilier , «Contributions of the Environmental Scanning Electron Microscope and X-ray diffraction in investigating the structural evolution of a $\mathrm{SiO} 2$ Aggregate attacked by Alkali-Silica Reaction », Journal of Materials Science, 39,20, 6221-6226, (2004).

11. G. N.Barbosa and H. P.Oliveira, Synthesis and characterization of $\mathrm{V} 2 \mathrm{O} 5-\mathrm{SiO} 2$ xerogel composites prepared by base catalysed sol-gel method,J. Non-Cryst Solids, 352, 3009-3014, (2006)

12. Z. L.Chen and P. Shen, Thermally activated sintering-coarsening-coalescence-

polymerization of amorphous silica nanoparticles, J. Ceramics International, 39, 2365-2373, (2012)

13. M.G. Garnica-Romo, J.M. Yanez-Limon, M. Villicana, J.F. Perez-Robles, R. Zamorano-Ulloa and J. Gonzalez-Hernandez, Structural evolution of sol-gel SiO2 heated glasses containing silver particles,J. Phys. Chem. Solids,65, 1045-1052, (2004).

14. P. Schmidt and F. Fröhlich, Temperature dependent crystallographic transformations in chalcedony, $\mathrm{SiO} 2$, assessed in mid infrared spectroscopy,J. Spectrochimica Acta A 78, 1476-1481, (2011).

15. H. El Rassy and A.C. Pierre, NMR and IR spectroscopy of silica aerogels with different hydrophobic characteristics,J. Non-Cryst Solids, 351, 1603-1610, (2005). 